\title{
Application of Imperialist Competitive Algorithm to Solve Constrained Economic Dispatch
}

\author{
Ghasem Mokhtari ${ }^{1}$, Ahmad Javid Ghanizadeh ${ }^{1^{*}}$, Esmaeil Ebrahimi ${ }^{1}$ \\ ${ }^{1}$ Electrical Engineering Department, Amirkabir University of Technology, Tehran, Iran \\ ghanizadeh@aut.ac.ir
}

\begin{abstract}
In the constrained dynamic ED problem, considering some practical operation constraints of generators, such as ramp rate limits and prohibited operating zones, electric power generation of units are scheduled. So, this paper, considering these constraints, presents a new optimization technique based on Imperialist Competitive Algorithm (ICA) to solve the Economic Dispatch (ED) problem in power systems. To show the efficiency of the proposed method, this algorithm is applied to solve constrained dynamic ED problem of two power systems with 6 and 15 units. The results are compared to those achieved from conventional methods such as Simulated Annealing (SA), Genetic Algorithm (GA) and Particle Swarm Optimization (PSO). The experimental results show that the proposed method is capable to determine the solutions of ED problem more fast and accurate than other conventional approaches.
\end{abstract}

Keywords: Economic Dispatch, Optimization, Imperialist Competitive Algorithm (ICA)

\section{Introduction}

One of the fundamental cases in power system operation is Economic Dispatch (ED) problem. It should be an optimization problem to reduce the operation cost of units while satisfying constraints. Two types of methods are used to optimize the generation output of units. The first type of methods employed various mathematical programming techniques. These conventional methods include the lambda-iteration method, the base point and participation factors method, the gradient method and etc. [1-3]. To use these kinds of methods for solving ED problem, the main assumption is that the incremental cost curves of the units are increased linearly. However, in practical systems considering the nonlinear characteristics of generators, this assumption is not acceptable. In addition, in a large system the methods based on mathematical programming have oscillatory problem [4].

Noted methods (mathematical methods) usually used to solve one type of ED problem known as static ED problem. The second type of ED problem is known as dynamic ED problem.

The objective function of dynamic ED is to determine generator output considering power balance, generating unit capacity limits, prohibited operating zones, valve point effect and multiple fuel cost. To solve the second type of ED problem (dynamic ED problem), random search optimization methods, such as Evolutionary Programming (EP) [5], Hybrid Immune Genetic Algorithm (HIGA) [6], Tabu Search (TS) [7] and Particle Swarm Optimization (PSO) [4] have been used.

Most of the random search optimization methods have two important problems:

1. Trapping in local minima.

2. Slow rate of convergence.

Received: January $11^{\text {st }}$, 2012. Accepted: November $29^{\text {th }}, 2012$ 
To prevent these kinds of problem, this paper proposes the use of a new evolutionary algorithm known as Imperialist Competitive Algorithm (ICA) to solve dynamic ED problem.

The ICA is a meta-heuristic optimization method that is based on modeling of the attempts of countries to dominate other courtiers. Like other evolutionary ones, this algorithm starts with an initial population. Populations are in two types: colonies and imperialists that all together form some empires. Imperialistic competition among these empires forms the basis of this algorithm. During this competition, weak empires collapse and powerful ones take possession of their colonies. Imperialistic competition converges to a state in which there exists only one empire and its colonies are the same position and have the same cost as the imperialist [8]. In [9] ICA is used for static ED problem. In this paper, this algorithm is applied to solve the constrained ED problem.

The rest of this paper organized as follow:

The mathematic formulation of the dynamic ED problem is presented in section II. The ICA is introduced in section III and the implementation of this algorithm for ED problem is explained in section IV. Finally, in section V, the proposed method is verified by using two different power systems with 6 and 15 units.

\section{Problem Formulation}

The dynamic ED problem is reviewed here for completeness and to clarify the constraints that are observed.

The objective function of the dynamic ED problem is to schedule the output of generation units to meet the required demand at minimum operating cost while satisfying all units' constraints. So, it can be said as follow:

The objective function of the ED problem can be expressed as [10]:

$$
\min F_{t}=\sum_{i=1}^{n} F_{i}\left(P_{i}\right)=\sum_{i=1}^{n}\left(a_{i}+b_{i} P_{i}+c_{i} P_{i}^{2}\right)
$$

where $F_{t}$ is the total operation cost; $F_{i}$ is the generation cost function of the $i$ th generator, $a_{i}, b_{i}$ and $c_{i}$ are the coefficients of cost function of the $i$ th generator; $P_{i}$ is the output power of the $i$ th generator and $n$ is the number of generators.

The main equality constraint is the constraint of power balancing which can be expressed as follow:

$$
\sum_{i=1}^{n} P_{i}=P_{D}+P_{L}
$$

where $P D$ is the load demand and $P L$ is the total transmission losses, which is a function of the unit power outputs that can be represented as follow:

$$
P_{L}=\sum_{i=1}^{n} \sum_{j=1}^{n} P_{i} B_{i j} P_{j}+\sum_{i=1}^{n} B_{0 i} P_{i}+B_{00}
$$

where $B$ is matrix that depends on transmission lines parameters.

To achieve the actual economic operation, two inequality constraints of generator are taken into account [10].

Firstly, the ramp rate restrictions of generators are caused in view of the fact that the thermal generating outputs cannot be adjusted quickly. To consider the operating process, the ramp rate limits are included in the ED problem to make sure the feasibility of the solutions.

As a result, the inequality constraints because of ramp rate limits for unit generation changes are presented as follow: 


$$
\max \left(P_{i}^{\min }, P_{i}^{0}-D R_{i}\right) \leq P_{i} \leq \min \left(P_{i}^{\max }, P_{i}^{0}+U R_{i}\right)
$$

where $P_{i}^{0}$ is the previous output power, $P_{i}^{\min }$ and $P_{i}^{\max }$ are the minimum and maximum outputs of the $i$ th generator, respectively. $U R i, D R i$ are the up ramp and down ramp limits of the $i$ th generator.

Secondly, the prohibited operating zones in the performance curve of a typical thermal unit are because of steam valve operation or fluctuation in a shaft bearing [11]. Practically, the shape of the input-output curve in the neighborhood of the prohibited zone is difficult to be determined by actual performance testing or operating records. In the actual operation, adjusting the generation output $P_{i}$ of a unit must avoid the unit operating in the prohibited zones. The feasible operating zones of unit $i$ can be presented as follows:

$$
P_{i} \in\left\{\begin{array}{l}
P_{i}^{\min } \leq P_{i} \leq P_{i, 1}^{l} \\
P_{i, j-1}^{u} \leq P_{i} \leq P_{i, j}^{l} \quad j=2,3, \ldots, n_{i} \\
P_{i, n_{i}}^{u} \leq P_{i} \leq P_{i}^{\max }
\end{array}\right.
$$

where $n_{i}$ is the number of prohibited zones of the $i$ th generator. $P_{i, j}{ }^{l} ; P_{i, j}{ }^{u}$ are the lower and upper power output of the prohibited zones $j$ of the $i$ th generator, respectively.

\section{Imperialist Competitive Algorithm}

Imperialism is the policy of spreading the power of an imperial beyond its own boundaries. An imperialist dominate other countries by direct rule or by less obvious means such as control of market for goods or raw materials.

The Imperialist Competitive Algorithm (ICA) was proposed by Esmaeil and Caro [8], this method is a new socio-politically motivated global search strategy that has recently been introduced for dealing with different optimization tasks. In [10], this algorithm is used to find the optimized weights of artificial neural network. Moreover, [12, 13] design an optimal controller using this algorithm.

Figure 1 shows the flow chart of the ICA [8]. Like the same evolutionary algorithms, ICA commences with an initial population of $P$ countries which are generated randomly within the feasible space. Each country is shown by country ${ }_{i}=\left[p_{1}, p_{2}, \ldots, p_{N v a r}\right]$ which $N_{v a r}$ is dimension of optimization problem. The best countries in the initial population, considering the cost function of them, are selected as the imperialists and other counties known as the colonies of these imperialists.

To build initial empires, colonies are divided among imperialists Based on imperialist's power. To divide the colonies among imperialists proportionally, the following normalized cost of an imperialist is defined:

$$
C_{n}=c_{n}-\max \left(c_{i}\right)
$$

Where $c_{n}$ is the cost of nth imperialist and $C_{n}$ is the normalized cost. As a result the normalized power of each imperialist can be defined:

$$
p_{n}=\left|\frac{C_{n}}{\sum_{i=1}^{N_{\text {imp }}} C_{i}}\right|
$$


Ghasem Mokhtari, et al.

After dividing colonies among imperialists, these colonies start closing to their empire. The total power of an empire can be determined by the power of imperialist country plus a percentage of power of its colonies as follow:

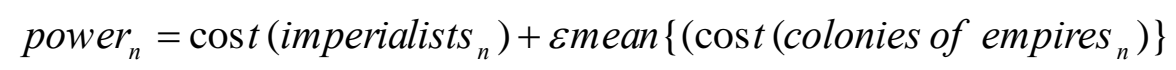

The way by which the colonies move toward empires can be found in [8].

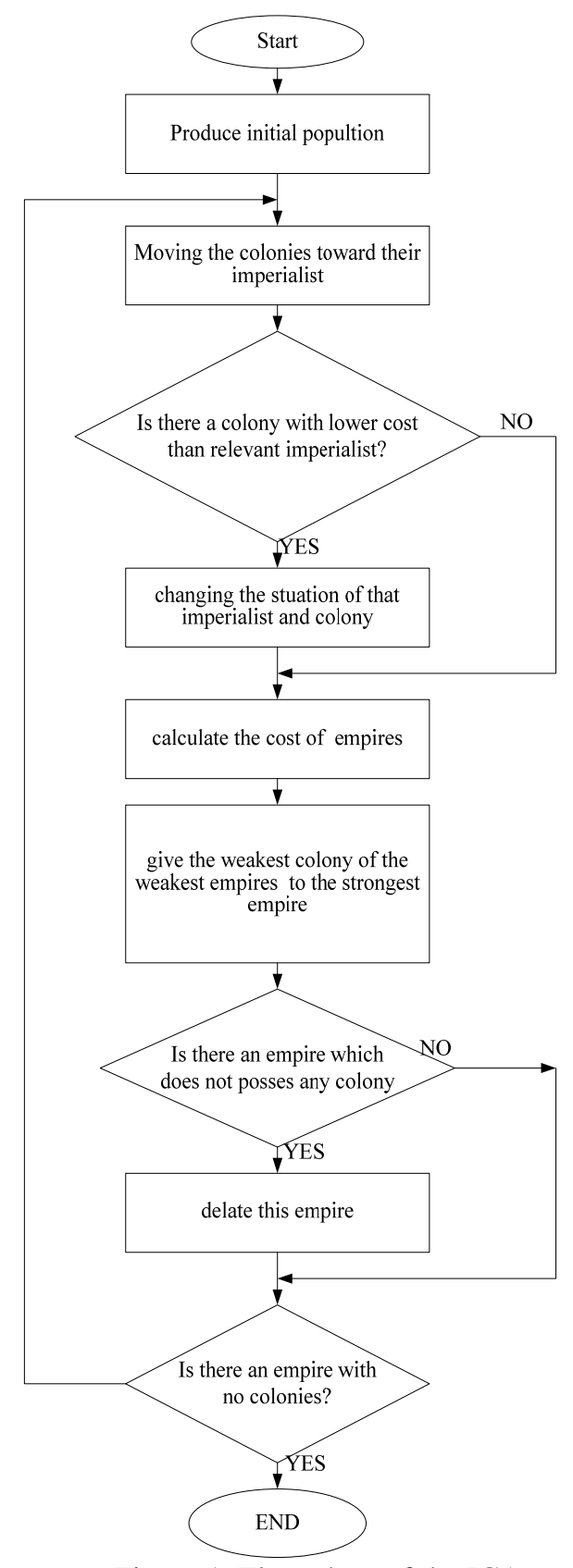

Figure 1. Flow chart of the ICA 
Where power $_{n}$ is the total power (cost) of the $n$th empire and $\xi$ is a positive number less than 1. Considering [8], its amount is set to 0.1 .

After starting competition, any empire that cannot succeed in this competition and cannot increase its power will be eliminated from the competition. The results of competition will be an increase in the power of strong empires and a decrease in the power of weak ones. With Jassing computation, Weak empires will lose their power and they will collapse. At the end, jy using the movement of colonies toward their relevant imperialist and also the collapse nechanism, one imperial will exit which is the answer of optimization problem.

\section{Application Of ICA To ED Problem}

In this section the implementation of the ICA for solving dynamic ED problem is described.

To apply this algorithm to ED problem, two mechanisms should be specified.

A. Initialization and structure of solutions

B. Constraints handling

These two mechanisms can be used as follow in ICA to determine optimized output of generating units in ED problem.

\section{A. Initialization and structure of solutions}

In the initialization process, several initial solutions are created randomly for the ICA. If the initial population of the ICA (countries) considered as the output generation value of units $\left(P_{j 1}{ }^{0}, P_{j 2}{ }^{0}, \ldots, P_{j n}{ }^{0}\right) . j=1, \ldots . ., m$. where $m$ is the number of countries in ICA and $n$ is the number of generators. To create a set of solutions, equality and inequality constraints should be satisfied. So, the procedure as follow is applied to ICA.

Step (1)

read system data

Step (2) the boundary of feasible solution is defined as

follow:

$$
\begin{aligned}
& P_{i_{\text {_lower }}}=\max \left(P_{i}^{\min }, P_{i}^{0}-D R_{i}\right) \\
& P_{i_{\text {_upper }}}=\min \left(P_{i}^{\max }, P_{i}^{0}+U R_{i}\right)
\end{aligned}
$$

Step (3) for $\mathrm{i}=1: \mathrm{n}$

$$
P_{i}=P_{i_{-} \text {lower }}+\operatorname{rand}() \times\left(P_{i_{-} \text {upper }}-P_{i_{-} \text {lower }}\right)
$$

End

where $\operatorname{rand}()$ is a uniform random value in the range $[0,1]$.

\section{B. Constraints handling with ICA}

In evolutionary optimization algorithm, handling the existing equality and inequality constraints is very important. In literatures, different methods for handling constraints in evolutionary computation optimization algorithms are presented. The most commonly used constraints handling methods among them are: preserving feasible solution method, infeasible solution rejection method, penalty function method and solution repair method [13]. In this paper the last two methods are used as follow to handle constraint in ICA.

To handle equality constraints in equation (2), penalty function method used as [4]. The evaluation function is adopted as (6), it is reciprocal of the generation cost function $F_{\text {cost }}$ and power balance constraints $P_{p b c}$ as in (7) and (8).

$$
f=\frac{1}{F_{\text {cost }}+P_{p b c}}
$$

where, 


$$
\begin{gathered}
F_{\text {cost }}=1+a b s\left(\frac{\left(\sum_{i=1}^{n} F_{i}\left(P_{i}\right)-F_{\min }\right)}{\left(F_{\max }-F_{\min }\right)}\right. \\
P_{p b c}=1+\left(\sum_{i=1}^{n} P_{i}-P_{D}-P_{L}\right)^{2}
\end{gathered}
$$

$F_{\max }$ maximum generation cost among all individuals in the initial population;

$F_{\min }$ minimum generation cost among all individuals in the initial population.

Moreover, to satisfy the inequality constraint of prohibited zone, solution repair method is used. To prevent solution to fall in the prohibited zone we do as follow:

Step (1): for all units specify prohibited zones (suppose $\left[a_{i}{ }^{k}, b_{i}{ }^{k}\right]$ as the $k$ th prohibited zone of unit $i$ )

Step (2): $\quad$ for each unit

$$
\begin{aligned}
& \text { If } \quad a_{i}^{k}<P_{i}<\frac{\left(a_{i}^{k}+b_{i}^{k}\right)}{2} \\
& P_{i}=a_{i}^{k}+\left(a_{i}^{k}-b_{i}^{k-1}\right) \times \operatorname{rand}() \\
& \text { Else if } \quad \frac{\left(a_{i}^{k}+b_{i}^{k}\right)}{2}<P_{i}<b_{i} \\
& P_{i}=a_{i}^{k}+\left(a_{i}^{k+1}-b_{i}^{k}\right) \times \operatorname{rand}() \\
& \text { End }
\end{aligned}
$$

\section{Case Studies}

To verify the effectiveness of the ICA for ED problem, two different power systems with 6 and 15 units were tested. Moreover, a comparison is made with SA, GA, HIGA, PSO, TS and MTS that are listed in $[4,6,10]$. The software like in [10] is implemented by the MATLAB language on a on a laptop computer with Core2Duo $2.5 \mathrm{GHz}$ CPU and 2 GByte RAM.

According to the experience, the following parameters are used in the ICA.

Number of countries $=80$

Number of imperialist $=8$

Maximum iteration $=30$

Revolution Rate $=0.2$

Damp Ratio $=0.99$

Uniting Threshold $=0.02$

Moreover, the parameters of the other optimization methods are listed in [23].

\section{A. 6- generating unit system}

The system contains 6 units, 26 buses and 46 lines [4]. The characteristics of the units are listed in Table I. The load demand is 1263 MW. In normal operation of the system, the loss coefficients B matrix with 100 MVA base capacity is shown in [4].

Table 1. Generation unit data of six units system

\begin{tabular}{c|ccccccccc}
\hline & $\begin{array}{c}P_{i}^{0} \\
(M W)\end{array}$ & $\begin{array}{c}P_{i}^{\min } \\
(M W)\end{array}$ & $\begin{array}{c}P_{i}^{\max }(M W) \\
(\$)\end{array}$ & $\begin{array}{c}a_{i} \\
(\$ / M W)\end{array}$ & $\begin{array}{c}c_{i} \\
\left(\$ / M W^{2}\right)\end{array}$ & $\begin{array}{c}U R_{i} \\
(M W / h)\end{array}$ & $\begin{array}{c}D R_{i} \\
(M W / h)\end{array}$ & $\begin{array}{c}\text { Prohibited } \\
\text { zones }(M W)\end{array}$ \\
\hline 1 & 440 & 100 & 500 & 240 & 7.0 & 0.0070 & 80 & 120 & {$[210,240]$} \\
2 & 170 & 50 & 200 & 200 & 10.0 & 0.0095 & 50 & 90 & $\begin{array}{c}{[350,380]} \\
{[90,110]} \\
3\end{array}$ \\
& 200 & 80 & 300 & 220 & 8.5 & 0.0090 & 65 & 100 & $\begin{array}{c}{[140,160]} \\
{[150,170]} \\
{[210,240]} \\
4\end{array}$ \\
& 150 & 50 & 150 & 200 & 11.0 & 0.0090 & 50 & 90 & $\begin{array}{c}{[80,90]} \\
{[110,120]} \\
5\end{array}$ \\
& 190 & 50 & 200 & 220 & 10.5 & 0.0080 & 50 & 90 & $\begin{array}{c}90,110] \\
{[140,150]} \\
{[75,85]} \\
6\end{array}$ \\
& 110 & 50 & 120 & 190 & 12.0 & 0.0075 & 50 & 90 & {$[100,105]$} \\
\hline
\end{tabular}


In $[6,10]$ six methods (MTS, PSO, HIGA TS, GA and SA) were employed to test on this system. Considering these results and the result of proposed method, Table II elaborates the best solutions of all methods, which satisfy the practical system constraints such as the ramp rate limits and prohibited zones of units. It can be clearly seen in Table II that the solution of the proposed method is the second one which has the minimum cost among all methods. With scheduling the output power of units correctly by the proposed method, the losses of system is decreased; therefore, total cost of system is reduced.

In addition, in Table 3, the statistical results of all methods such as maximum and minimum generation cost, best generation cost and standard deviation are shown. These results are determined after 30 trials.

Table 2. Best solution of six units system

\begin{tabular}{c|cccccc|c}
\hline Unit power output & HIGA [6] & SA [10] & GA [10] & TS [10] & PSO [10] & MTS [10] & ICA \\
\hline$P_{1}(M W)$ & 447.399 & 478.1258 & 462.0444 & 459.0753 & 447.5823 & 448.1277 & $\mathbf{4 3 7 . 9 1 4 2}$ \\
$P_{2}(M W)$ & 173.241 & 163.0249 & 189.4456 & 185.0675 & 172.8387 & 172.8082 & $\mathbf{1 7 6 . 5 6 9 7}$ \\
$P_{3}(M W)$ & 263.382 & 261.7143 & 254.8535 & 264.2094 & 261.3300 & 262.5932 & $\mathbf{2 6 5 . 0 0 0 0}$ \\
$P_{4}(M W)$ & 138.980 & 125.7665 & 127.4296 & 138.1222 & 138.6812 & 136.9605 & $\mathbf{1 5 0 . 0 0 0 0}$ \\
$P_{5}(M W)$ & 165.392 & 153.7056 & 151.5388 & 154.4716 & 169.6781 & 168.2031 & $\mathbf{1 4 7 . 8 7 7 2}$ \\
$P_{6}(M W)$ & 87.052 & 93.7965 & 90.7150 & 74.9900 & 85.8963 & 87.3304 & $\mathbf{9 7 . 7 5 5 2}$ \\
Total output & 1275.446 & 1276.1339 & 1276.0270 & 1275.94 & 1276.0066 & 1276.0232 & $\mathbf{1 2 7 5 . 1 1 6 3}$ \\
(MW) & 12.446 & 13.1317 & 13.0268 & 12.9422 & 13.0066 & 13.0205 & $\mathbf{1 2 . 1 1 6 8}$ \\
$P_{\text {loss }}(\mathrm{MW})$ & 15443.1 & 15461.10 & 15457.96 & 15454.89 & 15450.14 & 15450.06 & $\mathbf{1 5 4 4 9 . 0 6}$ \\
Total cost $(\$ / \mathrm{h})$ & & & & & & & \\
\hline
\end{tabular}

Table 3. Comparison of ICA performance with other methods for 6 units system

\begin{tabular}{c|cccc}
\hline Methods & Maximum cost $(\$ / \mathrm{h})$ & Average cost $(\$ / \mathrm{h})$ & Minimum cost $(\$ / \mathrm{h})$ & Standard deviation \\
\hline SA [10] & 15545.50 & 15488.98 & 15461.10 & 28.3678 \\
GA [10] & 15524.69 & 15477.71 & 15457.96 & 17.4072 \\
TS [10] & 15498.05 & 15472.56 & 15454.89 & 13.7195 \\
PSO [10] & 15491.71 & 15465.83 & 15450.14 & 10.1502 \\
MTS [10] & 15453.64 & 15451.17 & 15450.06 & 0.9287 \\
ICA & $\mathbf{1 5 4 9 1 . 7 1}$ & $\mathbf{1 5 4 5 2 . 0 5}$ & $\mathbf{1 5 4 4 9 . 0 6}$ & $\mathbf{2 . 0 4 5 8}$ \\
\hline
\end{tabular}

\section{B. 15-generating unit system}

The second system to verify the proposed method contains 15 units. The characteristics of units are listed in Table 4. In this system, the load demand is fixed at $2630 \mathrm{MW}$. Moreover, the loss coefficient $B$ matrices are shown in [4].

Table 4. Generation unit data of 15 units system

\begin{tabular}{|c|c|c|c|c|c|c|c|c|c|}
\hline Unit & $P_{i}^{0}(M W)$ & $P_{i}^{\min }(M W)$ & $P_{i}^{\max }(M W)$ & $a_{i}(\$)$ & $b_{i}(\$ / M W)$ & $\begin{array}{c}c_{i} \\
\left(\$ / M W^{2}\right) \\
\end{array}$ & $\begin{array}{c}U R_{i} \\
(M W / h) \\
\end{array}$ & $\begin{array}{c}D R_{i} \\
(M W / h) \\
\end{array}$ & $\begin{array}{c}\text { Prohibited zones } \\
(M W) \\
\end{array}$ \\
\hline 1 & 400 & 150 & 455 & 671 & 10.1 & 0.000299 & 80 & 120 & - \\
\hline 2 & 300 & 150 & 455 & 574 & 10.2 & 0.000183 & 80 & 120 & $\begin{array}{l}{[180,225][305,} \\
335][420,450]\end{array}$ \\
\hline 3 & 105 & 20 & 130 & 374 & 8.8 & 0.001126 & 130 & 130 & - \\
\hline 4 & 100 & 20 & 130 & 374 & 8.8 & 0.001126 & 130 & 130 & $\begin{array}{c}{[180,200][305,} \\
335][390,420]\end{array}$ \\
\hline 5 & 90 & 150 & 470 & 461 & 10.4 & 0.000205 & 80 & 120 & $\begin{array}{c}{[230,255][365,} \\
395][430,455]\end{array}$ \\
\hline 6 & 400 & 135 & 460 & 630 & 10.1 & 0.000301 & 80 & 120 & - \\
\hline 7 & 350 & 135 & 465 & 548 & 9.8 & 0.000364 & 80 & 120 & - \\
\hline 8 & 95 & 60 & 300 & 227 & 11.2 & 0.000338 & 65 & 100 & - \\
\hline 9 & 105 & 25 & 162 & 173 & 11.2 & 0.000807 & 60 & 100 & - \\
\hline 10 & 110 & 25 & 160 & 175 & 10.7 & 0.001203 & 60 & 100 & - \\
\hline 11 & 60 & 20 & 80 & 186 & 10.2 & 0.003586 & 80 & 80 & - \\
\hline 12 & 40 & 20 & 80 & 230 & 9.9 & 0.005513 & 80 & 80 & {$[30,40][55,65]$} \\
\hline 13 & 30 & 25 & 85 & 225 & 13.1 & 0.000371 & 80 & 80 & - \\
\hline 14 & 20 & 15 & 55 & 309 & 12.1 & 0.001929 & 55 & 55 & - \\
\hline 15 & 20 & 15 & 55 & 323 & 12.4 & 0.004447 & 55 & 55 & - \\
\hline
\end{tabular}


After simulating this case, the best solutions obtained from all methods that satisfy the system constraints are shown in Table 5. It can be clearly seen that the proposed method has the cheaper solution among the all methods.

In addition, after performing 30 trials, the statistical results of all method are listed in Table 6 . The results of Table 6 show that in spite of increasing the size of system, the proposed method is acceptable and truthful.

Table 5. Best solution of six units system

\begin{tabular}{c|cccccc}
\hline Unit power output & SA [10] & GA [10] & TS [10] & PSO [10] & MTS [10] & ICA \\
\hline$P_{1}(M W)$ & 453.6646 & 445.5619 & 453.5374 & 454.7167 & 453.9922 & $\mathbf{4 5 3 . 8 9 2 2}$ \\
$P_{2}(M W)$ & 377.6091 & 380.0000 & 371.9761 & 376.2002 & 379.7434 & $\mathbf{3 7 9 . 8 4 0 5}$ \\
$P_{3}(M W)$ & 120.3744 & 129.0605 & 129.7823 & 129.5547 & 130.0000 & $\mathbf{1 2 9 . 4 5 1 2}$ \\
$P_{4}(M W)$ & 126.2668 & 129.5250 & 129.3411 & 129.7083 & 129.9232 & $\mathbf{1 2 9 . 9 2 3 2}$ \\
$P_{5}(M W)$ & 165.3048 & 169.9659 & 169.5950 & 169.4407 & 168.0877 & $\mathbf{1 6 9 . 0 8 7 7}$ \\
$P_{6}(M W)$ & 459.2455 & 458.7544 & 457.9928 & 458.8153 & 460.0000 & $\mathbf{4 5 9 . 0 0 5 1}$ \\
$P_{7}(M W)$ & 422.8619 & 417.9041 & 426.8879 & 427.5733 & 429.2253 & $\mathbf{4 2 8 . 1 5 8 4}$ \\
$P_{8}(M W)$ & 126.4025 & 97.8230 & 95.1680 & 67.2834 & 104.3097 & $\mathbf{9 8 . 3 0 9 7}$ \\
$P_{9}(M W)$ & 54.4742 & 54.2933 & 76.8439 & 75.2673 & 35.0358 & $\mathbf{4 2 . 0 3 5 8}$ \\
$P_{10}(M W)$ & 149.0879 & 144.2214 & 133.5044 & 155.5899 & 155.8829 & $\mathbf{1 5 5 . 6 3 6 2}$ \\
$P_{11}(M W)$ & 77.9594 & 77.3002 & 68.3087 & 79.9522 & 79.8994 & $\mathbf{7 9 . 9 0 2 3}$ \\
$P_{12}(M W)$ & 73.9489 & 77.0371 & 79.6815 & 79.8947 & 79.9037 & $\mathbf{8 0 . 0 0 0 0}$ \\
$P_{13}(M W)$ & 25.0022 & 31.1537 & 28.3082 & 25.2744 & 25.0220 & $\mathbf{2 5 . 1 8 9 1}$ \\
$P_{14}(M W)$ & 16.0636 & 15.0233 & 17.7661 & 16.7318 & 15.2586 & $\mathbf{1 5 . 1 5 6 7}$ \\
$P_{15}(M W)$ & 15.0196 & 33.6125 & 22.8446 & 15.1967 & 15.0796 & $\mathbf{1 5 . 1 2 9 8}$ \\
Total output $(\mathrm{MW})$ & 2663.29 & 2661.23 & 2661.53 & 2661.19 & 2661.36 & $\mathbf{2 6 6 0 . 7 1 7 2}$ \\
$P_{\text {loss }}(\mathrm{MW})$ & 33.2737 & 31.2363 & 31.4100 & 31.1697 & 31.3523 & $\mathbf{3 0 . 7 5 4 9}$ \\
Total cost $(\$ / \mathrm{h})$ & 32786.40 & 32779.81 & 32762.12 & 32724.17 & 32716.87 & $\mathbf{3 2 7 1 2 . 3 4}$ \\
\hline
\end{tabular}

Table 6. Comparison of ICA performance with other methods for 15 units system

\begin{tabular}{c|ccccc}
\hline Methods & $\begin{array}{c}\text { Maximum } \\
\text { cost }(\$ / \mathrm{h})\end{array}$ & $\begin{array}{c}\text { Average cost } \\
(\$ / \mathrm{h})\end{array}$ & $\begin{array}{c}\text { Minimum cost } \\
(\$ / \mathrm{h})\end{array}$ & $\begin{array}{c}\text { Standard } \\
\text { deviation }\end{array}$ & $\begin{array}{c}\text { Average CPU } \\
\text { time }(\mathrm{s})\end{array}$ \\
\hline SA & 33028.95 & 32869.51 & 32786.40 & 112.32 & 71.25 \\
GA & 33041.64 & 32841.21 & 32779.81 & 81.22 & 48.17 \\
TS & 32942.71 & 32822.84 & 32762.12 & 60.59 & 26.41 \\
PSO & 32841.38 & 32807.45 & 32724.17 & 21.24 & 13.25 \\
MTS & 32796.15 & 32767.21 & 32716.87 & 17.51 & 3.65 \\
ICA & $\mathbf{3 2 7 9 4 . 0 5}$ & $\mathbf{3 2 7 5 9 . 8 7}$ & $\mathbf{3 2 7 1 2 . 3 4}$ & $\mathbf{1 5 . 4 5}$ & $\mathbf{1 . 5 8}$ \\
\hline
\end{tabular}

\section{Computation efficiency}

Table 7 shows the computational time of the proposed method for both case studies. It can be seen that proposed method has enough efficiency for solving ED problem. In addition, Figure 2, shows the convergence characteristic of the proposed method for 15- generating unit system. It can be seen that the proposed method after 9 iterations reaches to the optimum point. The best method among other methods, as shown in [10], it reaches to the optimum point after around 85 iterations. So, the convergence of the proposed method to the optimum solution is faster than the other methods

Table 7. Computational time of the proposed method

\begin{tabular}{l|l}
\hline & Average CPU time (s) \\
\hline 6- generating unit system & 0.58 \\
15-generating unit system & 2.21 \\
\hline
\end{tabular}




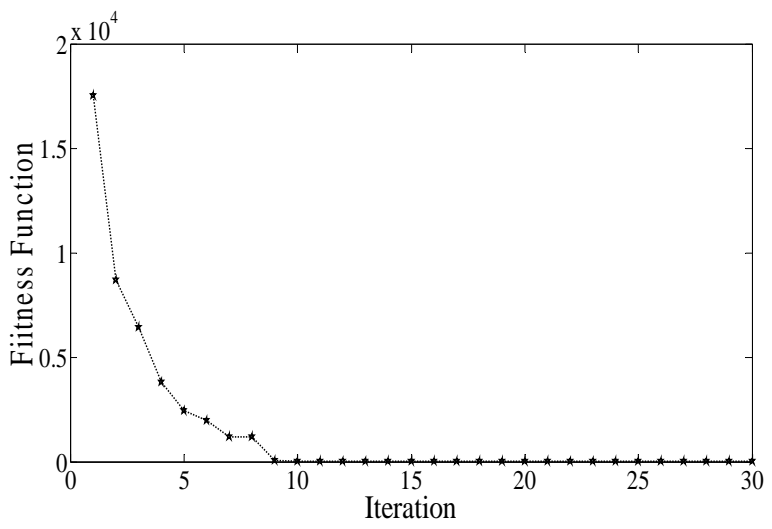

Figure 2. Convergence characteristic of the proposed method

\section{Conclusion}

In this paper Imperialist Competitive Algorithm (ICA) for solving the Economic Dispatch (ED) problem was presented. Two appropriate strategies for initialization and handling the equality and inequality constraints were utilized which always provide feasible solutions. To show the ability of the proposed method for ED problem, two different test systems are used. Comparing the results of the proposed method with other conventional methods that is listed in [10], it can be derived that the proposed method can find the best solution better than other methods. Moreover, the effectiveness of the proposed method is shown by low computational time and fast convergence.

\section{References}

[1] A. J. Wood, B. F. Wollenberg, "Power generation, operation and control," New York: John Wiley \& Sons, 1994.

[2] R. A. Jabr, A. H. Coonick and B. J. Cory, "A homogeneous linear programming algorithm for the security constrained economic dispatch problem," IEEE Transactions on Power Systems, vol. 15, no. 3, pp. 930-936, Aug 2000.

[3] S. D. Chen and J. F. Chen, "A direct Newton-Raphson economic emission dispatch," International Journal of Electrical Power \& Energy Systems, vol. 25, no. 5, pp. 411-417, Jun 2003.

[4] T. A. A. Victoire and A. E. Jeyakumar, "Discussion of particle swarm optimization to solving the economic dispatch considering the generator constraints," IEEE Transactions on Power Systems, vol. 19, no. 4, pp. 2121-2122, Nov 2004.

[5] M. A. Azad and E. Fernandes, "A modified differential evolution based solution technique for economic dispatch problems," Journal of Industrial and Management Optimization, vol. 8, no. 4, pp. 1017-1038, Nov 2012.

[6] A. Rabii, S. Mobaieen, B. Mohamady and A. Suroody, "A new heuristic algorithm for solving non-convex economic power dispatch," Journal of Applied Sciences, vol. 11, no. 23, pp. 3791-3796, 2011.

[7] W. Ongsakul, S. Dechanupaprittha, I. Ngamroo, "Parallel tabu search algorithm for constrained economic dispatch," IEE Proceedings- Generation, Transmission and Distribution, vol. 151, no. 2, pp. 157-166, 2004.

[8] E. Atashpaz-Gargari and C. Lucas, "Imperialist competitive algorithm: An algorithm for optimization inspired by imperialistic competition," IEEE Congress on Evolutionary Computation, CEC 2007, pp. 4661-4667, 25-28 Sept. 2007.

[9] H. Chahkandi Nejad and R. Jahani, "A new approach to economic load dispatch of power system using Imperialist Competitive Algorithm," Australian Journal of Basic and Applied Sciences, vol. 5, no. 9, pp. 835-843, 2011. 
[10] S. Pothiya, I. Ngamroo, W. Kongprawechnon, "Application of multiple tabu search algorithm to solve dynamic economic dispatch considering generator constraints," Energy Conversion and Management, vol. 49, pp. 506-516, Apr. 2008.

[11] P. H. Chen and H. C. Chang, "Large-scale economic dispatch by genetic algorithm," IEEE Transactions on Power Systems, vol. 10, no.4, pp. 1919-1926, Nov. 1995.

[12] M. Abdechiri, K. Faez, H. Bahrami, "Neural Network Learning Based on Chaotic Imperialist Competitive Algorithm," 2nd International Workshop on Intelligent Systems and Applications (ISA), pp. 1-5, 22-23 May 2010.

[13] E. Atashpaz-Gargari, F. Hashemzadeh, C. Lucas, "Designing MIMO PIID controller using colonial competitive algorithm: Applied to distillation column process," IEEE Congress on Evolutionary Computation, CEC 2008, (IEEE World Congress on Computational Intelligence), pp. 1929-1934, 2008.

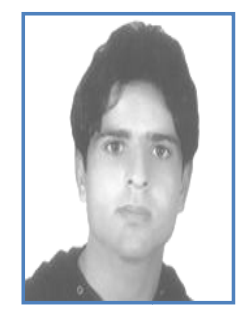

Ghasem Mokhtari was born in Mashhad. He received the B.S. degree in electrical engineering from Ferdowsi University of Mashhad in 2007. He is currently pursuing the M.S. degree in the electrical engineering department at the Amirkabir University of Technology (AUT), Tehran, Iran. His research interest include TEP problem, power market and power system optimization.

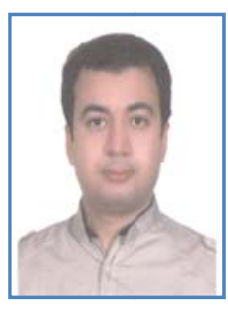

Ahmad Javid Ghanizadeh was born in Herat, Afghanistan, in 1981. He received his B.Sc. and M.Sc. in electrical engineering from Sahand University of Technology, Tabriz, Iran and Ferdowsi University of Mashhad, Mashhad, Iran, in 2004 and 2009, respectively. He is currently pursuing the Ph.D. degree in the electrical engineering department at the Amirkabir University of Technology (AUT), Tehran, Iran. His research interests include Power Quality, power system optimization and operation and transformers transients.

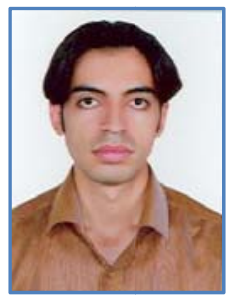

Esmaeil Ebrahimi was born in Maku, Iran, in 1987. He received the B. S. degree in electrical engineering from Tabriz University, Tabriz, Iran, in 2009. He is currently pursuing the M. S. degree in the Electrical Engineering department, Amirkabir University of Technology (AUT), Tehran, Iran. His research interests include distributed generation, power protection, and power electronics. 